\title{
Editorial: Zoonotic Microorganisms and Spread of Acquired Polymyxin Resistance Determinants
}

\author{
Azucena Mora ${ }^{1 *}$, Maria Jorge Campos ${ }^{2}$ and Alberto Quesada ${ }^{3 *}$ \\ ${ }^{1}$ Laboratorio de Referencia de Escherichia coli, Dpto. de Microbioloxía e Parasitoloxía, Universidade de Santiago de \\ Compostela, Lugo, Spain, ${ }^{2}$ Marine and Environmental Sciences Centre, School of Tourism and Maritime Technology (ESTM), \\ Polytechnic of Leiria, Peniche, Portugal, ${ }^{3}$ Departamento de Bioquímica, Facultad de Veterinaria, Universidad de \\ Extremadura, Cáceres, Spain
}

Keywords: colistin resistance, enterobacteria, zoonotic agent, antimicrobial resistance (AMR), MCR genes

\section{Editorial on the Research Topic}

\section{Zoonotic Microorganisms and Spread of Acquired Polymyxin Resistance Determinants}

\section{OPEN ACCESS}

Edited and reviewed by: Rustam Aminov,

University of Aberdeen, United Kingdom

${ }^{*}$ Correspondence: Azucena Mora

azucena.mora@usc.es

Alberto Quesada

aquesada@unex.es

Specialty section:

This article was submitted to Antimicrobials, Resistance and Chemotherapy,

a section of the journal

Frontiers in Microbiology

Received: 05 January 2022 Accepted: 06 January 2022 Published: 04 February 2022

Citation:

Mora A, Campos MJ and Quesada A (2022) Editorial: Zoonotic Microorganisms and Spread of Acquired Polymyxin Resistance Determinants.

Front. Microbiol. 13:849316 doi: $10.3389 /$ fmicb.2022.849316
Currently, the use of colistin as a last-resort antibiotic in human medicine is highly compromised due to the continuous emergence of resistant enterobacteria with acquired determinants like plasmid-mediated resistance genes $(m c r)$, mutations that activate the PmrAB system, or still unknown mechanisms. On the other hand, the correlation between the individual, or combined activity of these mechanisms, with the functional expression of resistance to colistin is still poorly understood. Based on a One-Health approach, this topic aimed to gather knowledge in the transmission and expression of colistin resistance determinants in Gram-negative bacteria isolated from different sources.

Since the $m c r-1$ plasmid-mediated colistin resistance gene was discovered in China in 2015 (Liu et al., 2016), numerous surveys have been performed worldwide to monitor the prevalence and spread of $\mathrm{mcr}$ in food animals. Industrial farming, particularly poultry and swine, have been highlighted as a zoonotic source of $m c r$ (mainly $m c r-1$ ) Escherichia coli isolates (Irrgang et al., 2016; García-Meniño et al., 2018). Cheng et al. analyzed 200 fecal swabs collected from six swine farms in northeastern China between July 2016 and June 2017. A total of 176 E. coli strains were isolated and the prevalence of $m c r-1$ in colistin-resistant E. coli was 53.33\% (56/105). $m c r-1$-positive E. coli showed extensive antimicrobial resistance (AMR) profiles with the presence of additional resistance genes, increased expression of multidrug efflux pump-associated genes, and increased biofilm formation ability. The results of growth assay, competition experiment, and plasmid stability testing showed that acquisition of $m c r-1$-harboring plasmids could reduce the fitness of bacterial hosts, but $m c r-1$ remained stable in the recipient strain.

The availability of accurate systems for the diagnosis of AMR is a cornerstone both for its clinical study and for the investigation of the propagation of clones with low susceptibility to colistin. Zhu et al. analyzed the performance of three mainstream commercial antimicrobial susceptibility testing (VITEK 2 $\mathbb{R}$ COMPACT, Phoenix ${ }^{\mathrm{TM}} \mathrm{M} 50$, and Bio-kont) in parallel to standard broth microdilution, finding out slight but significant differences among them, especially for enterobacteria like Escherichia spp., Klebsiella spp., and Citrobacter spp. Retrospective studies on frozen stored collections of samples are being used to investigate the evolution of new threats, like plasmid-mediated colistin resistance determinants. Miguela-Villoldo et al. showed that an SYBR Green qPCR assay designed to detect $m c r-1$ in pig caecum samples is the best option to provide a highly representative frame of the initial population present in the sample, and although the freezethaw process affects bacterial viability, culture-based methods might be a useful complement to study colistin resistance levels. 
Poultry and poultry meat have also been highlighted as important contributors to the global antimicrobial burden with potential transmission risk for consumers (Díaz-Jiménez et al., 2021). In Lebanon, where the first $m c r$-1-positive E. coli found in poultry dates to 2015, Hiba Al-Mir et al. report a prevalence of $84.4 \% \mathrm{mcr}$ - 1 positive poultry farms across three Lebanese governorates. The study was conducted on poultry samples collected in 2018 in one large slaughterhouse and originated from 32 individual farms. Furthermore, numerous associated resistances were identified, including the presence of $b l a_{C T X-M}$ or bla $a_{C M Y}$ genes, and the $m c r-1$ gene was mostly located in IncX4 $(n=36)$ and IncI2 $(n=24)$ plasmids.

The findings of Nakano et al. in Japan uncover the potential circulation of $m c r-1$ mediated colistin-resistant E. coli among livestock and farmers. The authors analyzed fecal samples from 295 healthy livestock (202 cattle and 93 swine) and 62 healthy livestock farmers (53 cattle farmers and nine swine farmers) collected between 2013 and 2015, from 72 livestock farms. The prevalence of $m c r-1$-harboring E. coli was 25 (8.47\%) of the 295 livestock from 11 farms, and 3.77\% (2/53 strains) for cattle farmers, and $11.11 \%$ ( $1 / 9$ strains) for swine farmers. Considering the isolates obtained from livestock and farmers, in four farms, nine isolates had the same genotypical characteristics (sequence types and pulsed-field gel electrophoresis band patterns), plasmid characteristics (incompatibility group and plasmid transferability), and minimum inhibitory concentrations.
Due to the high number of colonized animals, slaughterhouses might represent a significant source of the introduction of $\mathrm{mcr}$ genes into the food chain through possible contamination of carcasses and products. Furthermore, these bacteria might accumulate in process waters and wastewater from slaughterhouses, contributing to a broad spread of the resistance to other environmental ecosystems including surface waters. The study of Savin et al. supports the necessity of implementing advanced wastewater treatment technologies to limit the contamination of the environment with bacteria expressing resistances against last resort antimicrobials. The authors performed a study to evaluate the occurrence of colistin-resistant Enterobacteriaceae in process waters and wastewater from two poultry and two pig slaughterhouses in Germany. Their findings demonstrated a high occurrence of colistin-resistant $E$. coli and Klebsiella pneumoniae carrying mcr-1 on transferable plasmids (incompatibility groups IncI1, IncHI2, IncX4, IncF, and IncI2) in poultry and pig slaughterhouses and indicate their dissemination into surface water. Environmental contamination with colistin-resistant enterobacteria carrying $\mathrm{mcr}$ genes is also described by Lopes et al., who detected the ST131 clone of $E$. coli isolated from a kale crop in Brazil. In addition to $\mathrm{mcr}-1$, bla $a_{C T X}-M-15$, and $q n r B 19$ genes were associated with $\mathrm{IncH} 12$, IncF, and ColE1 replicons, respectively, the two first harboring additional AMR determinants and being efficiently transferred by conjugation.

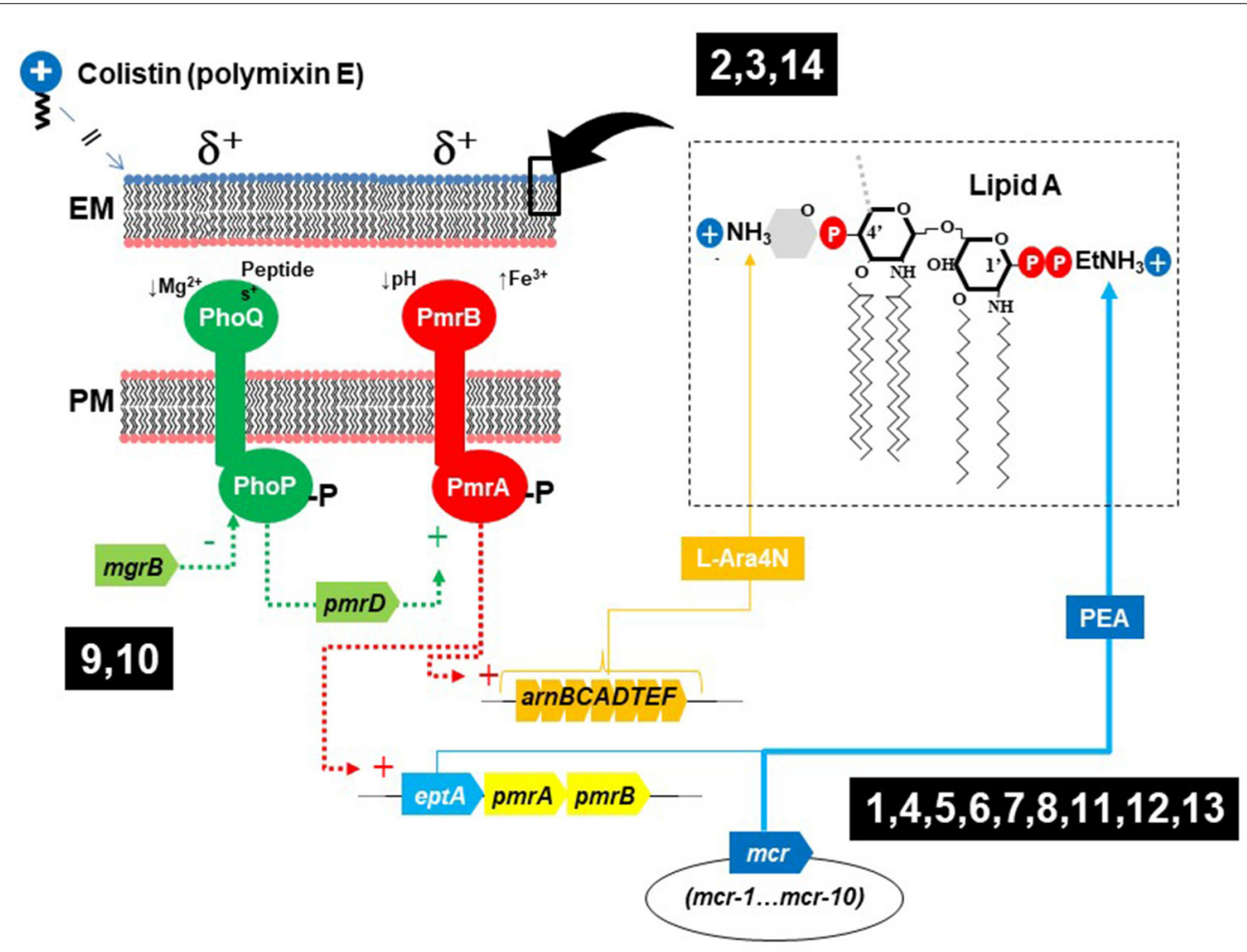

FIGURE 1 | Elements involved in colistin resistance in enterobacteria, biological context of the articles published in this research topic. (1, Cheng et al.; 2, Zhu et al.; 3 , Miguela-Villoldo et al.; 4, Hiba Al-Mir et al.; 5, Nakano et al.; 6, Savin et al.; 7, Lopes et al.; 8, Zhang et al.; 9, Ngbede et al.; 10, Azam et al.; 11, Hu et al.; 12, Vázquez et al.; 13, Diaconu et al.; 14, Panta and Doerrler). 
Wild birds play a key role in the spread of AMR due to their environmental exposure through ingested food or polluted water. In this respect, the results of Zhang et al. provide evidence that migratory birds are potential transmitters of AMR. The authors identified $22 \mathrm{mcr}$-1-positive E. coli from 303 Anser indicus fecal samples (7.3\%) collected in the Guangdong province of China. Coexisting with 24 other types of AMR genes, $m c r-1$ was located on IncX4, IncI2, and IncP plasmids.

Together with colistin, carbapenems are used as last-resort antibiotics. Ngbede et al. investigated carbapenem and colistin resistance in 583 non-duplicate Enterobacteriaceae isolates recovered from humans, animals, and the environment in Nigeria to find that $18.9 \%$ were resistant to at least one carbapenem and 9.1\% exhibited concurrent carbapenem-colistin resistance. No carbapenem-resistant isolates carried any known carbapenemase-producing gene. Whole-genome sequencing supported that concurrent carbapenem-colistin resistance was mediated by novel or previously described alterations in chromosomal efflux regulatory genes, particularly $m g r B$ (M1V), ompC (M1_V24del), ompK37 (I70M, I128M), ramR $(\mathrm{M} 1 \mathrm{~V})$, and $\operatorname{marR}(\mathrm{M} 1 \mathrm{~V})$. In addition, alterations/mutations were detected in the etpA, arnT, $c c r B, p m r B$ in colistinresistant bacteria and ompK36 in carbapenem-resistant bacteria. In contrast, Azam et al. found that among 11 colistin-resistant $K$. pneumoniae isolated from humans in India, present non-synonymous potentially deleterious mutations in the phoP, phoQ, pmrA, pmrB, and $m g r B$ genes, and amongst those, one clone carried the New Delhi metallo-beta-lactamase 1 gene (NDM-1) and expressed carbapenem resistance.

Salmonella is one of the leading causes of global bacterial food poisoning worldwide. However, the occurrence and transmission of $\mathrm{mcr}$ genes in Salmonella are still poorly understood. $\mathrm{Hu}$ et al. analyzed 755 foodborne Salmonella from 26 provinces in mainland China in 2016. As a result, $10 \%$ of the isolates were defined as multidrug-resistant (MDR), and two carried mcr-1: S. Derby CFSA231 and S. Typhimurium CFSA629. Both expressed an MDR phenotype and included a single circular chromosome and one plasmid. Among the 22 AMR genes identified in S. Derby CFSA231, only the $m c r-1$ gene was localized on the IncX4 type plasmid pCFSA231 while 20 chromosomal AMR genes, including four plasmid-mediated quinolone resistance (PMQR) genes, were mapped within a $64 \mathrm{~kb}$ Salmonella genomic island (SGI) like region. S. Typhimurium CFSA629 possessed 11 resistance genes including an mor-1.19 variant and two ESBL genes. The contribution by Vázquez et al. shows that $2.2 \%$ of foodborne Salmonella isolated in Asturias, Spain, between 2014 and 2019 were resistant to colistin. Four of these isolates, belonging to the European monophasic ST34 clone of $S$. Typhimurium characterized by chromosomal genes conferring resistance to ampicillin, streptomycin, sulfonamides, tetracycline, heavy metals, and arsenic \pm mercury, carried $\mathrm{mcr}$ 1 in IncX4 or IncHI2 plasmids. Moreover, Diaconu et al. reported that $6.2 \%$ of AMR isolates from foodstuffs belonging to the same ST34 clone presented the $m c r-9$ gene carried by IncHI 2 megaplasmids of near $300 \mathrm{~Kb}$, associated with increased resistance to colistin.

Previously unknown determinants for colistin resistance are starting to be revealed. In addition to chromosomal mutations affecting the two-component sensory systems (TCSS) PhoPQ or PmrAB, and the $m c r$ genes, modification of lipopolysaccharide lipid A with aminoarabinose (L-Ara4N) is carried out by the enzymes encoded in arn operons (Figure 1). These modifications might confer colistin resistance upon up-regulation by chromosomal mutations of TCSS and can also be plasmid-mediated, similarly to $\mathrm{mor}$ genes (Gallardo et al., 2021). Some Gram-negative bacteria are intrinsically resistant to very high levels of colistin, and the work by Panta and Doerrler evidenced that a DedA family protein in Burkholderia thailandensis (DbcA; DedA of Burkholderia required for colistin resistance) is a membrane transporter required for resistance to colistin, which function is partially restored by overexpression of arn genes or by the increase in membrane potential that can occur by lowering the $\mathrm{pH}$.

Published contributions on this topic are indicated in Figure 1 in relation to their context on colistin resistance (1, Cheng et al.; 2, Zhu et al.; 3, Miguela-Villoldo et al.; 4, Hiba Al-Mir et al.; 5, Nakano et al.; 6, Savin et al.; 7, Lopes et al.; 8, Zhang et al.; 9, Ngbede et al.; 10, Azam et al.; 11, $\mathrm{Hu}$ et al.; 12, Vázquez et al.; 13, Diaconu et al.; 14, Panta and Doerrler).

\section{AUTHOR CONTRIBUTIONS}

All authors listed have made a substantial, direct, and intellectual contribution to the work and approved it for publication.

\section{FUNDING}

Work in the laboratory of AQ is funded by the Spanish Ministry of Science and Innovation (AEI, Spain), the Junta de Extremadura, and FEDER (Grants PID2020-118405RBI00, IB20181, and grupo CTS059). AM acknowledges funds from the Agencia Estatal de Investigación (AEI, Spain), the Xunta de Galicia, and FEDER (Grants PID2019-104439RBC21/AEI/10.13039/501100011033 and ED431C 2021/11). Work in the laboratory of MC is funded by the Portuguese Foundation for Science and Technology (FCT) through the strategic project UID/04292/2020 granted to MARE-Marine and Environmental Sciences Centre.

\section{ACKNOWLEDGMENTS}

The guest editors very much appreciate all the authors, reviewers, and editorial members for their contributions to this Research Topic. 


\section{REFERENCES}

Díaz-Jiménez, D., García-Meniño, I., Herrera, A., Lestón, L., and Mora, A. (2021). Microbiological risk assessment of turkey and chicken meat for consumer: significant differences regarding multidrug resistance, $\mathrm{mcr}$ or presence of hybrid aEPEC/ExPEC pathotypes of E. coli. Food control. 2021:107713. doi: 10.1016/j.foodcont.2020.107713

Gallardo, A., Iglesias, M. R., Ugarte-Ruiz, M., Hernández, M., Miguela-Villoldo, P., Gutiérrez, G., et al. (2021). Plasmid-mediated Kluyvera-like arnBCADTEF operon confers colistin (hetero)resistance to Escherichia coli. Antimicrob Agents Chemother. 65, e00091-21. doi: 10.1128/AAC.00091-21

García-Meniño, I., García, V., Mora, A., Díaz-Jiménez, D., Flament-Simon, S. C., Alonso, M. P., et al. (2018). Swine enteric colibacillosis in Spain: pathogenic potential of mcr-1 ST10 and ST131 E. coli isolates. Front. Microbiol. 9:2659. doi: $10.3389 /$ fmicb.2018.02659

Irrgang, A., Roschanski, N., Tenhagen, B. A., Grobbel, M., SkladnikiewiczZiemer, T., Thomas, K., et al. (2016). Prevalence of mcr-1 in E. coli from livestock and food in Germany, 2010-2015. PLoS ONE 11:e0159863. doi: 10.1371/journal.pone.0159863

Liu, Y. Y., Wang, Y., Walsh, T. R., Yi, L. X., Zhang, R., Spencer, J., et al. (2016). Emergence of plasmid-mediated colistin resistance mechanism
MCR-1 in animals and human beings in China: a microbiological and molecular biological study. Lancet Infect. Dis. 16, 161-168. doi: 10.1016/S1473-3099(15)00424-7

Conflict of Interest: The authors declare that the research was conducted in the absence of any commercial or financial relationships that could be construed as a potential conflict of interest.

Publisher's Note: All claims expressed in this article are solely those of the authors and do not necessarily represent those of their affiliated organizations, or those of the publisher, the editors and the reviewers. Any product that may be evaluated in this article, or claim that may be made by its manufacturer, is not guaranteed or endorsed by the publisher.

Copyright (C) 2022 Mora, Campos and Quesada. This is an open-access article distributed under the terms of the Creative Commons Attribution License (CC BY). The use, distribution or reproduction in other forums is permitted, provided the original author(s) and the copyright owner(s) are credited and that the original publication in this journal is cited, in accordance with accepted academic practice. No use, distribution or reproduction is permitted which does not comply with these terms. 